\title{
Implications of recent progress in heavy-quark hadro-production
}

\author{
Maria Vittoria Garzelli* \\ II. Institute for Theoretical Physics, University of Hamburg \\ E-mail: maria.vittoria.garzelliedesy.de
}

\section{Sven-Olaf Moch}

II. Institute for Theoretical Physics, University of Hamburg

E-mail: sven-olaf.moch@desy.de

\section{Guenter Sigl}

II. Institute for Theoretical Physics, University of Hamburg

E-mail: guenter.sigledesy.de

We discuss recent theoretical progress in heavy-quark hadro-production, in particular focusing on processes involving charm-quarks, and on their implications in different fields of particle phenomenology, from collider to astroparticle physics.

12th International Symposium on Radiative Corrections (Radcor 2015) and LoopFest XIV (Radiative Corrections for the LHC and Future Colliders)

15-19 June, 2015

UCLA Department of Physics \& Astronomy Los Angeles, USA

\footnotetext{
${ }^{*}$ Speaker.

${ }^{\dagger}$ Work supported by the Deutsche Forschungsgemeinschaft in Sonderforschungsbereich 676
} 


\section{Introduction}

The production of heavy quarks, i.e., of quarks with a mass well above the QCD scale parameter $\Lambda_{Q C D}$, was investigated since early days of QCD and collider physics. The existence of a fourth quark, the charm, was theorized in the sixties (see, e.g., Refs. [1,2]) and confirmed in 1974 by the observation of the $\mathrm{J} / \Psi$ meson both at the Stanford Linear Accelerator Center [3] and at the Brookhaven National Laboratory [4]. The existence of a third family of quarks was postulated in 1973 [5] and confirmed by the E288 experiment at Fermilab, which observed bottomonium states [6] in 1977, and by the CDF and D0 collaborations at Tevatron, which in 1995 discovered the top quark [7,8]. The main difference between the relatively heavy (charm and bottom) and really heavy top-quark relies on their masses and on their lifetimes [9]. In particular, the top-quark decays well before hadronizing, whereas charm and bottom, although being, like the top, typically produced in perturbative processes (due to the fact that, like $m_{t}$, even $m_{c}$ and $m_{b} \gg \Lambda_{Q C D}$ ), are always observed as intermediate hadronic states ( $D$ and $B$ hadrons), and subsequently decay in lighter ones eventually accompanied by prompt leptons. As a consequence, the study of top-quark hadro-production, involving intermediate top state reconstruction from $b$-jets and additional leptons, missing energy, or light-jets, allows to get important information on the "hard" core of hadron-hadron collision processes at energies above the top production threshold, with hard-scatterings described by perturbation theory, and became possible only recently, i.e., at colliders with high enough center-of-mass energies. On the other hand, studies of bottom and charm-quarks performed since early days of accelerator physics with different beams have allowed not only for important tests of the validity of QCD and of the existence of new physics, but even to gain important information on the hadronization process [10].

Nowadays, most of the theoretical efforts at colliders concentrate on perturbative QCD, and the main focus of the heavy-quark working groups active at the most modern collider experiment, the Large Hadron Collider (LHC) at CERN, is the study of top-quark properties. In this respect, the hadronic pair-production of top-quarks is the most prominent signal, with tools for inclusive cross-section predictions up to NNLO QCD available [11,12]. At the differential level, first studies at NNLO for the Tevatron [13] and at the LHC [14] have appeared, and are actually necessary to meet the statistical accuracy reached during latest runs at the LHC. Additionally, recent theoretical developments have allowed to get predictions beyond $(\mathrm{N}) \mathrm{LO}$ also for different channels, ranging from single top to $t \bar{t}$ hadro-production in association with other particles (one or more vector bosons, the Higgs scalar, or jets). As for single top, NNLO QCD corrections at the fully differential level have been computed for the $t$-channel [15]. On the other hand, as for processes involving three or more final state particles at the parton level, the state-of-the-art is represented by NLO QCD predictions matched to Parton Shower (PS) approaches [16-24], eventually accompanied by procedures for merging states characterized by different light-jet multiplicities at the parton level $[25,26]$. Recently, additional efforts have also been devoted to the inclusion of the electroweak (EW) corrections, first of all at fixed order [27,28], with general frameworks matching NLO QCD + EW corrections to PS and EW showers under development.

Although LHC is often considered as a top-quark factory [29], one has to take into account that the cross-sections for bottom and charm hadro-production are even larger, making worth even the study of processes involving the direct production of these quarks, without passing through inter- 
mediate top states. In particular, for $p p$ collisions at $\sqrt{s}=13 \mathrm{TeV}$, the inclusive cross sections scale as $\sigma_{c \bar{c}} \sim \mathscr{O}(20) \sigma_{b \bar{b}} \sim \mathscr{O}(20.000) \sigma_{t \bar{t}}$ at NNLO in QCD. Although selected results from ATLAS and CMS are also available, charm and bottom-quark hadro-production is being explored in particular at $\mathrm{LHCb}$, which has provided distributions for heavy meson and baryon hadro-production at the fully differential level $[30,31]$, together with results on correlations in the production of heavymeson pairs [32]. Charm and bottom production measurements are useful for better understanding the composition of the proton (an example is given in section 3), and the running behavior of the strong coupling constant $\alpha_{S}$.

Furthermore, the experimental extraction of the values of the masses of heavy quarks and their connection with theory, taking into account that quark masses act as fundamental parameters in the Lagrangian of the Standard Model, has attracted a lot of interest and efforts, but presents difficulties. In case of top-quarks, long-standing discussions concern the relation between the experimentally extracted mass and a rigorously defined theoretical mass [33]. On the other hand, in case of charm and bottom quark, besides the complications inherent the description of the transition between the perturbative regime and the non-perturbative one acting in the hadronization, the main theoretical issue is represented by the slow convergence of the perturbative series for quark masses in the onshell scheme. This is particularly serious in case of charm, for which recent calculations pointed out the absence of convergence at 4-loop [34].

A recent review on top-quarks can be found, e.g., in Ref. [35]. In the following, we will concentrate in particular on the study of charm-quark hadro-production, by presenting some of the implications of theoretical predictions and experimental results at LHC, for astroparticle physics.

\section{Charm hadro-production cross-section}

Theoretical predictions for the total $c \bar{c}$ hadro-production cross-section including NNLO QCD radiative corrections have been presented in Ref. [36] for a wide range of energies and compared to experimental data from fixed target experiments, RHIC and LHC. These predictions have been obtained by an extension of the Hathor framework [11], originally designed to compute $t \bar{t}$ hadroproduction cross-sections. The comparison of the NNLO predictions with the NLO ones turned out to show a good perturbative convergence even at energies far larger that those reached at LHC, and allowed to estimate $K$-factors related to NNLO/NLO ratios and to compare them with the more commonly used $K$-factors for the NLO versus LO predictions. An example of typical cross-section values and related $K$-factors is shown in Fig. 1 . The NNLO $K$-factor is smaller than the NLO one over the whole range of considered energies for both the scale choices $\mu_{R}=\mu_{F}=m_{c}$ and $\mu_{R}=\mu_{F}=2 m_{c}$. Furthermore, while the first choice produces smaller $K$-factors at lower energies, the second choice leads to flatter $K$-factors, with a NNLO $K$-factor staying within 1.5 even at the highest energy considered here $\left(E_{p}=10^{10} \mathrm{GeV}\right.$ in the laboratory frame, equivalent to about $E_{C M} \sim 137 \mathrm{TeV}$ in the center-of-mass reference frame), where the $q g(\bar{q} g)$-channels dominates over the other initial-state partonic channels. The slope of the increase of the $K$-factor with $E_{p}$ at high energy is shaped by the small- $x$ behavior of the parton luminosity and the hard scattering cross sections. On the other hand, at low $E_{p}$, where all parton channels contribute at medium to large $x$ significantly, the charm-quark mass $m_{c}$ is no longer negligible and the shape of both the NNLO and NLO $K$-factors is sensitive to charm production threshold effects [37]. 

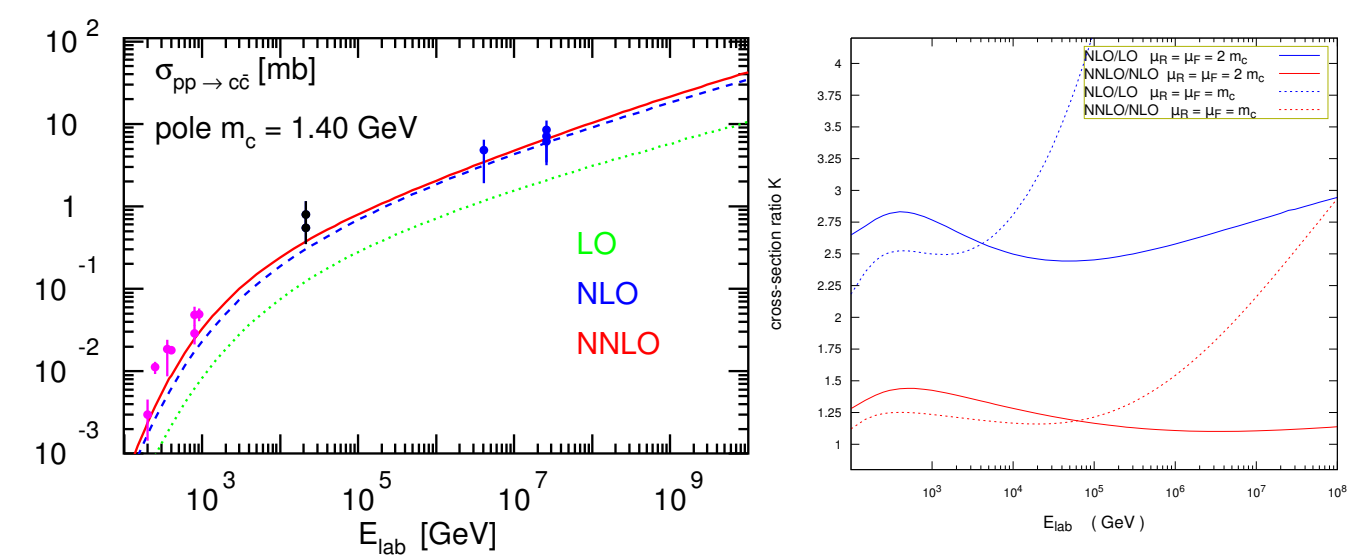

Figure 1: Total $\sigma_{p p \rightarrow c \bar{c}}$ as a function of the proton energy $E_{p}$ in the laboratory frame, including LO, NLO and NNLO QCD corrections, as compared to available experimental data from fixed target experiments [38], RHIC [39, 40], ALICE [41], ATLAS [42] and LHCb [30]. ABM11 PDFs at NNLO, $m_{c}=1.4 \mathrm{GeV}$ and $\mu_{R}=\mu_{F}=2 m_{c}$ are used as input of the theoretical calculation in the fixed flavour number scheme with $N_{f}=3$. The corresponding NNLO/NLO and NLO/LO K-factors are shown in the right panel (solid lines), together with those corresponding to the scale choice $\mu_{R}=\mu_{F}=m_{c}$ (dotted lines).

At the differential level, predictions for theoretical distributions for $c \bar{c}$ hadro-production are not yet available at NNLO. The state of the art is represented in this case by NLO QCD approaches matched to PS. In some of the available approaches and tools, EW corrections can be included as well. However, taking into account that these corrections are in general expected to be smaller than typical uncertainties due to renormalization and factorization scale variation, we neglect electroweak effects in the following. Theoretical predictions can be validated by comparison with experimental data from ALICE, ATLAS and LHCb. In particular, LHCb measured at both $\sqrt{s}=7 \mathrm{TeV}$ and $13 \mathrm{TeV}$ differential cross-sections for $D$-mesons in both transverse momentum and rapidity, considering the rapidity range $2 \leq y_{0} \leq 4.5$, corresponding to mid-peripheral collisions. An example of comparisons of the experimental data at $7 \mathrm{TeV}$ with the predictions by POWHEGBOX [43] + PYTHIA 6.4.128 [44] using the ABM11 [45] central PDF set at NLO, $\mu_{R}=\mu_{F}=\mu_{0}=\sqrt{p_{T, c}^{2}+4 m_{c}^{2}}$ and a charm-quark pole mass parameter $m_{c}=1.4 \mathrm{GeV}$ (for further discussion concerning the choice of these input see Ref. [36]), is presented in Fig. 2, showing consistency of theory with data even in the largest rapidity bins: experimental data turned out to always lie within the theory uncertainty bands due to scale and mass variation, obtained by considering the interval $m_{c} \in[1.25-1.55] \mathrm{GeV}$ and the $\left(\mu_{R}, \mu_{F}\right)$ combinations $\in[(2,2),(0.5,0.5)$, $(2,1),(1,2),(0.5,1),(1,0.5)] \mu_{0}$. Similar agreement between data and theory was found even for charged $D$-mesons.

\section{Implications of recent results of LHCb on Parton Distribution Functions}

Results of LHCb concerning charm and bottom hadro-production have been included in recent PDF fits, due to the complementarity of their coverage in Bjorken- $x$ with respect to that ensured by the data collected at HERA. In particular the HERA charm data allowed to probe the gluon $x$ region $10^{-4} \leq x \leq 10^{-1}$. On the other hand LHCb data, in particular those at large rapidity $(\sim 4-$ 4.5) allowed to extend the range on both sides, giving rise to a total $x$ coverage extending to the 

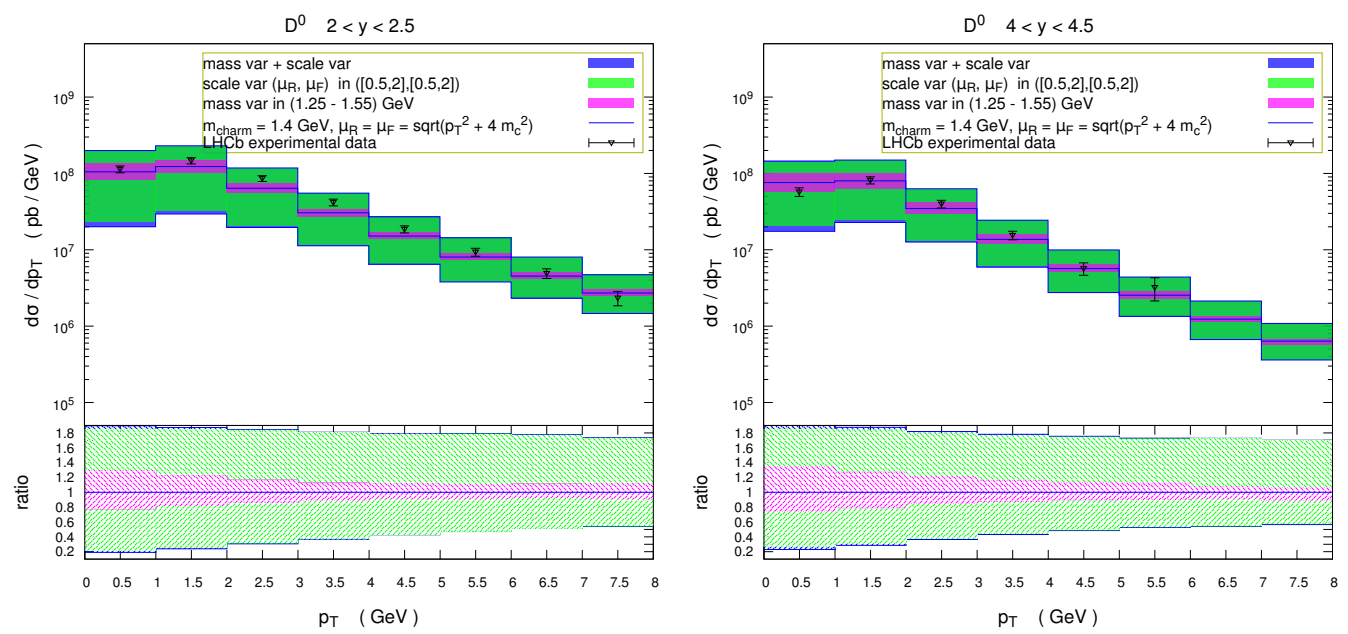

Figure 2: Theoretical predictions from POWHEGBOX + PYTHIA 6 for the $p_{T}$ distributions of $D^{0}$ mesons in $p p$ collisions at $7 \mathrm{TeV}$ vs. experimental data from LHCb. Scale and charm mass input are fixed as in Fig. 1.a. The NLO version of the ABM11 PDFs with $N_{f}=3$ is used. The violet and green band correspond respectively to charm mass and scale uncertainty, combined in quadrature in the blue band. The two panels correspond to the most central and the most peripheral rapidity bin explored at $\mathrm{LHCb}$, respectively.

interval $10^{-6} \lesssim x \lesssim 1$. This extension is especially important in case of high-energy and very-highenergy collisions: in fact the higher are the energies, the more asymmetric $g g$ initial states can come into play, characterized by $x_{1} \ll 1$ and $x_{2} \sim x_{F}$, with $x_{F}$ being the Feynman- $x$.

The first attempt to include LHCb data in PDF fits was performed by the PROSA collaboration [46], using LHCb data at $\sqrt{s}=7 \mathrm{TeV}$, in association with data from HERA to perform the fit, following a procedure similar to that originally used for performing the HERAPDF fit [47], further extended to the inclusion of hadron collider data. Both, the absolute LHCb data (i.e. $d^{2} \sigma / d p_{T} d y$ ) and the ratio of data $(\mathrm{d} \sigma / d y) /\left(d \sigma / d y_{0}\right)$ in each $p_{T}$ bin were fitted, with $d \sigma / d y_{0}$ being the crosssection in the central bin $3 \leq y_{0} \leq 3.5$ of the total measured rapidity range $2 \leq y_{0} \leq 4.5$.

The resulting PDF best-fits, together with the accompanying eigenvectors corresponding to model, fit and parameterization uncertainties, extended PDF grids down to $x \sim 10^{-6}$ and led to a reduced uncertainty for gluons in the region $10^{-6}<x<10^{-4}$ with respect to previous fits.

A second attempt was performed by members of the NNPDF collaboration, using LHCb data at $\sqrt{s}=7 \mathrm{TeV}$ and $13 \mathrm{TeV}$ to further constrain the most recent NNPDF fit. In particular, it turned out that the NNPDF3.0 fit [48] gives rise to uncertainty bands which open up dramatically for $x<10^{-4}$, where NNPDF3.0 PDFs are essentially unconstrained. Including LHCb data has led to a new fit, labeled as NNPDF3.0 + LHCb [49], with modifications of the shape of the central gluon density as a function of $x$ and reduced uncertainty band in the low $x$ region. Although this fit is supposed to represent an improvement with respect to the NNPDF3.0 PDF version nowadays widely used at colliders, it is not yet publicly available, at least as far as we know.

We also notice that the ABM11 and ABM12 [50] fits, although having been done well before and thus non including any $\mathrm{LHCb}$ data, for low- $x$ gluons turned out to have all their eigenvectors well included within the uncertainty bands of the PROSA fit and to present compatibility even with the NNPDF3.0 + LHCb uncertainty band. 


\section{Astrophysical implications of charm hadro-production}
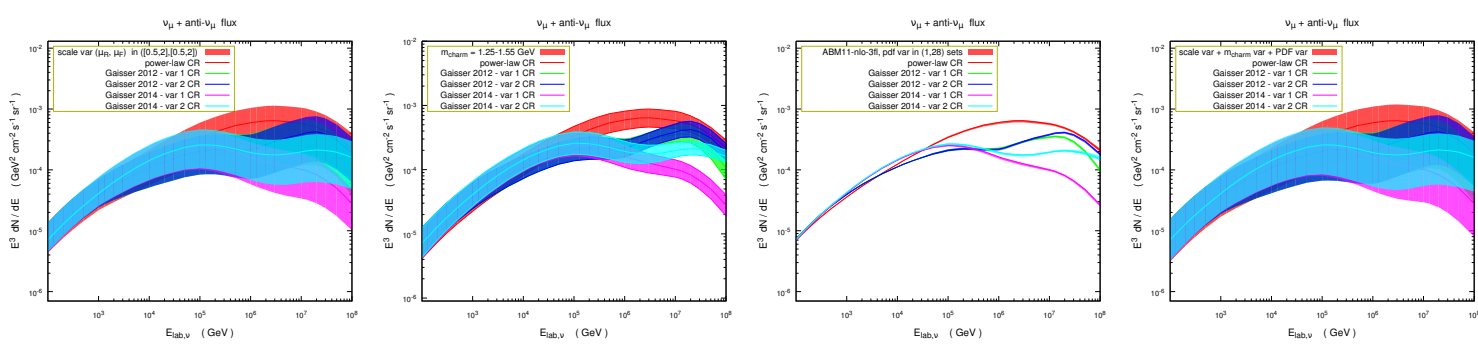

Figure 3: Prompt $\left(v_{\mu}+\bar{v}_{\mu}\right)$ fluxes as a function of $E_{v}$ for five different primary CR input fluxes: uncertainties due to $\mu_{R}$ and $\mu_{F}$ scale variation, $m_{c}$ variation and PDF variation (limited to the 28 eigenvectors of the ABM11 NLO PDF fit), together with their combination in quadrature are shown in panel a, b, c, d, respectively. QCD inputs are chosen as in Fig. 2.

The considerations made above on charm hadro-production in QCD find an interesting application and test-bed in astroparticle physics, in the problem of the evaluation of prompt neutrino fluxes [36]. In particular, it is well known that the bulk of atmospheric neutrinos is due to the leptonic and semi-leptonic decay of light mesons (mostly $\pi^{ \pm}$'s and $K^{ \pm}$'s) originated by the interaction of primary cosmic rays (CR) impinging into the Earth's atmosphere with the atmospheric nuclei [51]. This conventional neutrino flux, peaked at neutrino energies $E_{v}$ below $1 \mathrm{GeV}$ and known to fall down quite rapidly with $E_{v}$, is investigated since many years (see e.g., Ref. [52]). On the other hand, at higher energies, the IceCube collaboration has recently reported evidence for a leptonic flux extending at least up to the $\sim \mathrm{PeV}$ energy region [53,54], whose origin is still under discussion. Although it is believed that a large portion of this flux has an astrophysical origin, i.e., it is due to neutrinos reaching the Earth from deep space, traveling almost undeflected from far galactic or extra-galactic sources, a rigorous investigation has to consider and quantify which fraction of these neutrinos can instead originate in the Earth's atmosphere. The latter process would be possible at energies like those explored by IceCube, thanks to semi-leptonic decays of $D$-mesons and baryons, eventually formed in the interaction of CR primaries with the atmosphere. At energies high enough this process is more effective in producing neutrinos than the process of conventional production described above because highly boosted $\pi^{ \pm}$and $K^{ \pm}$have a suppressed probability to decay in neutrinos, while crossing the Earth's atmosphere, whereas semi-leptonic decays would still be abundant for $D$-mesons and baryons, having a larger mass, a smaller life-time $\left(\tau_{0, D} \sim 10^{-22} \mathrm{~s}\right.$ versus $\tau_{0, \pi} \sim 10^{-8} \mathrm{~s}$ ) and decaying just immediately after their formation. This neutrino component is thus called prompt component. We have computed it in QCD, by describing CR interactions with the Earth's atmosphere in terms of nucleon-nucleon $(N N)$ collisions and by using the same tools and input described in Section 2 as for the simulation of the $N N \rightarrow c \bar{c} \rightarrow D+X$ processes. In particular, predictions from POWHEGBOX + PYTHIA 6 in the same configuration validated with respect to $\mathrm{LHCb}$ data at $7 \mathrm{TeV}$ (see Fig. 1) were used even to compute the prompt neutrino flux presented in our paper [36]. More recently another group adopted a similar framework (POWHEGBOX + PYTHIA8 [55]) together with the NNPDF3.0 + LHCb PDFs, getting predictions for prompt neutrino fluxes as well [56], with a method very close to our one and same CR primary input fluxes. 

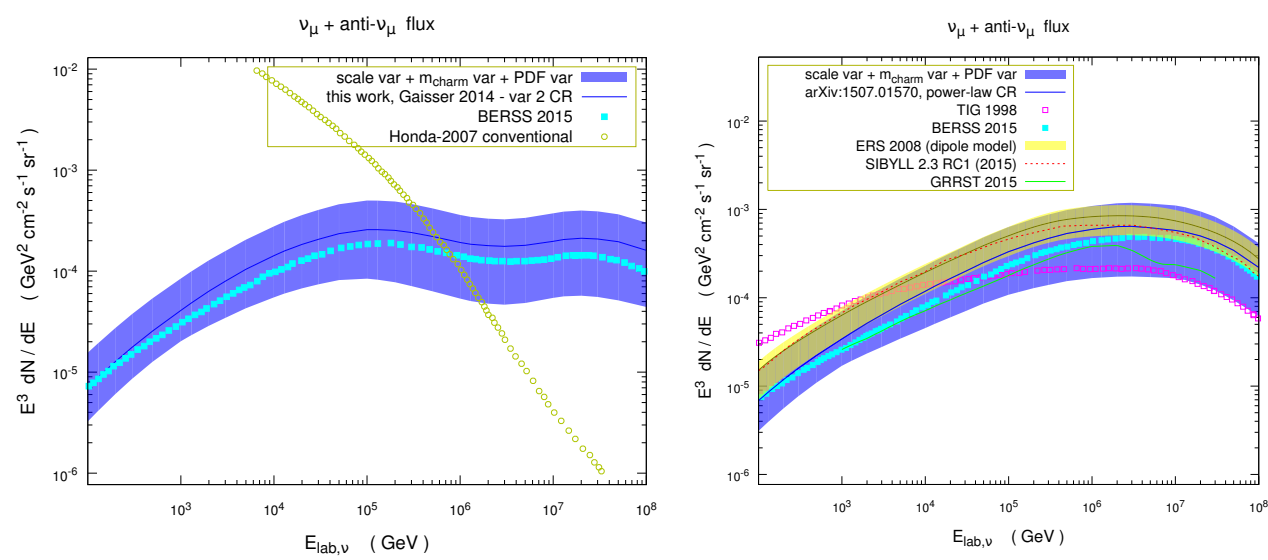

Figure 4: a) prompt $\left(v_{\mu}+\bar{v}_{\mu}\right)$ flux and its uncertainty vs. conventional flux for one of the Gaisser primary CR spectrum variants; b) a comparison between our predictions for the prompt flux (blue line and bands) and other recent predictions for the power-law CR primary spectrum. See text for more detail.

Our predictions, together with separate uncertainty bands due to scale, charm mass and PDF variation (the latter restricted to the 28 eigenvectors of the ABM11 NLO PDF fit), are shown in Fig. 3, for five different CR primary fits [57,58]. Actually, it is evident that at high energy, the astrophysical uncertainty related to our poor knowledge of primary CR fluxes becomes even more important than the QCD uncertainty. This means that if, on the one hand, these predictions can be further refined by a future improved QCD description of charm hadro-production including effects from higher order corrections capable of limiting/overcoming the huge scale uncertainty of the NLO computation, amounting to many ten percent, on the other hand, it is also indispensable to improve our knowledge of CR fluxes by means of forthcoming CR data from extended air shower experimental arrays, like e.g. the Pierre Auger Observatory.

The relative importance of conventional and prompt neutrino flux is presented in Fig. 4.a, showing a transition energy, i.e. the energy where the prompt flux overcomes the conventional one, around $E_{v, \text { trans }}=6_{-3}^{+12} \cdot 10^{5} \mathrm{GeV}$.

Finally, a comparison of our predictions with other old $[59,60]$ and recent predictions $[56,61$, 62] obtained during last year, is presented in Fig. 4.b, showing that all most recent predictions turn out to lie within our theoretical uncertainty band.

\section{References}

[1] B. Bjorken and S. L. Glashow, Elementary Particles and SU(4), Phys. Lett. 11 (1964) 255-257.

[2] S. L. Glashow, J. Iliopoulos, and L. Maiani, Weak Interactions with Lepton-Hadron Symmetry, Phys. Rev. D2 (1970) 1285-1292.

[3] SLAC-SP-017 Collaboration, J. E. Augustin et al., Discovery of a Narrow Resonance in e+ $e$ Annihilation, Phys. Rev. Lett. 33 (1974) 1406-1408. [Adv. Exp. Phys.5,141(1976)].

[4] 5598 Collaboration, J. J. Aubert et al., Experimental Observation of a Heavy Particle J, Phys. Rev. Lett. 33 (1974) 1404-1406.

[5] M. Kobayashi and T. Maskawa, CP Violation in the Renormalizable Theory of Weak Interaction, Prog. Theor. Phys. 49 (1973) 652-657. 
[6] S. W. Herb et al., Observation of a Dimuon Resonance at 9.5-GeV in 400-GeV Proton-Nucleus Collisions, Phys. Rev. Lett. 39 (1977) 252-255.

[7] CDF Collaboration, F. Abe et al., Observation of top quark production in p̄p collisions, Phys. Rev. Lett. 74 (1995) 2626-2631, [hep-ex/9503002].

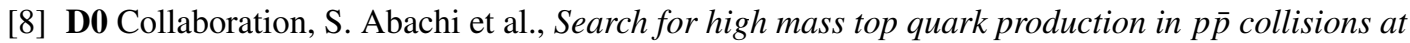
$\sqrt{s}=1.8$ TeV, Phys. Rev. Lett. 74 (1995) 2422-2426, [hep-ex/9411001].

[9] Particle Data Group Collaboration, K. Olive et al., Review of Particle Physics, Chin.Phys. C38 (2014) 090001.

[10] E. Norrbin and T. Sjostrand, Production and hadronization of heavy quarks, Eur. Phys. J. C17 (2000) 137-161, [hep-ph/0005110].

[11] M. Aliev, H. Lacker, U. Langenfeld, S. Moch, P. Uwer, and M. Wiedermann, HATHOR: HAdronic Top and Heavy quarks crOss section calculatoR, Comput. Phys. Commun. 182 (2011) 1034-1046, [arXiv:1007.1327].

[12] M. Czakon and A. Mitov, Top++: A Program for the Calculation of the Top-Pair Cross-Section at Hadron Colliders, Comput. Phys. Commun. 185 (2014) 2930, [arXiv:1112 . 5675].

[13] M. Czakon, P. Fiedler, D. Heymes, and A. Mitov, NNLO QCD predictions for fully-differential top-quark pair production at the Tevatron, arXiv:1601.05375.

[14] M. Czakon, D. Heymes, and A. Mitov, High-precision differential predictions for top-quark pairs at the LHC, arXiv: 1511.00549.

[15] M. Brucherseifer, F. Caola, and K. Melnikov, On the NNLO QCD corrections to single-top production at the LHC, Phys. Lett. B736 (2014) 58-63, [arXiv: 1404 . 7116].

[16] A. Kardos, C. Papadopoulos, and Z. Trocsanyi, Top quark pair production in association with a jet with NLO parton showering, Phys. Lett. B705 (2011) 76-81, [arXiv:1101.2672].

[17] M. V. Garzelli, A. Kardos, C. G. Papadopoulos, and Z. Trocsanyi, Standard Model Higgs boson production in association with a top anti-top pair at NLO with parton showering, Europhys. Lett. 96 (2011) 11001, [arXiv:1108.0387].

[18] M. V. Garzelli, A. Kardos, C. G. Papadopoulos, and Z. Trocsanyi, $t \bar{t} W^{+-}$and $t \bar{t} Z$ Hadroproduction at NLO accuracy in QCD with Parton Shower and Hadronization effects, JHEP 11 (2012) 056, [arXiv:1208.2665].

[19] A. Kardos and Z. Trócsányi, Hadroproduction of t-anti-t pair with two isolated photons with PowHel, Nucl. Phys. B897 (2015) 717-731, [arXiv: 1408 . 0278].

[20] M. V. Garzelli, A. Kardos, and Z. Trócsányi, Hadroproduction of t $\bar{t} b \bar{b}$ final states at LHC: predictions at NLO accuracy matched with Parton Shower, JHEP 03 (2015) 083, [arXiv: 1408 . 0266 ].

[21] F. Cascioli, P. Maierhöfer, N. Moretti, S. Pozzorini, and F. Siegert, NLO matching for $t \bar{t} b \bar{b}$ production with massive b-quarks, Phys. Lett. B734 (2014) 210-214, [arXiv:1309.5912].

[22] S. Höche, F. Krauss, P. Maierhöfer, S. Pozzorini, M. Schönherr, and F. Siegert, Next-to-leading order QCD predictions for top-quark pair production with up to two jets merged with a parton shower, Phys. Lett. B748 (2015) 74-78, [arXiv:1402.6293].

[23] J. Alwall, R. Frederix, S. Frixione, V. Hirschi, F. Maltoni, O. Mattelaer H. S. Shao, T. Stelzer, P. Torrielli, and M. Zaro, The automated computation of tree-level and next-to-leading order differential cross sections, and their matching to parton shower simulations, JHEP 07 (2014) 079, [arXiv:1405.0301]. 
[24] M. Czakon, H. B. Hartanto, M. Kraus, and M. Worek, Matching the Nagy-Soper parton shower at next-to-leading order, JHEP 06 (2015) 033, [arXiv: 1502 . 00925$].$

[25] R. Frederix and S. Frixione, Merging meets matching in MC@NLO, JHEP 12 (2012) 061, [arXiv:1209.6215].

[26] T. Gehrmann, S. Hoche, F. Krauss, M. Schonherr, and F. Siegert, NLO QCD matrix elements + parton showers in $e^{+} e^{-} \longrightarrow$ hadrons, JHEP 01 (2013) 144, [arXiv:1207.5031].

[27] S. Frixione, V. Hirschi, D. Pagani, H. S. Shao, and M. Zaro, Weak corrections to Higgs hadroproduction in association with a top-quark pair, JHEP 09 (2014) 065, [arXiv: 1407.0823 ].

[28] S. Frixione, V. Hirschi, D. Pagani, H. S. Shao, and M. Zaro, Electroweak and QCD corrections to top-pair hadroproduction in association with heavy bosons, JHEP 06 (2015) 184, [arXiv:1504.03446].

[29] T. Han, The 'Top Priority' at the LHC, Int. J. Mod. Phys. A23 (2008) 4107-4124, [arXiv:0804.3178].

[30] LHCb Collaboration, R. Aaij et al., Prompt charm production in pp collisions at sqrt(s)=7 TeV, Nucl. Phys. B871 (2013) 1-20, [arXiv:1302.2864].

[31] LHCb Collaboration, R. Aaij et al., Measurements of prompt charm production cross-sections in pp collisions at $\sqrt{s}=13 \mathrm{TeV}$, arXiv:1510.01707.

[32] LHCb Collaboration, R. Aaij et al., Observation of double charm production involving open charm in pp collisions at $\sqrt{s}=7 \mathrm{TeV}$, JHEP 06 (2012) 141, [arXiv: 1205.0975$].$

[33] S. Moch et al., High precision fundamental constants at the TeV scale, arXiv: 1405.4781.

[34] P. Marquard, A. V. Smirnov, V. A. Smirnov, and M. Steinhauser, Quark Mass Relations to Four-Loop Order in Perturbative QCD, Phys. Rev. Lett. 114 (2015), no. 14 142002, [arXiv: 1502.01030 ].

[35] V. del Duca and E. Laenen, Top physics at the LHC, Int. J. Mod. Phys. A30 (2015), no. 35 1530063, [arXiv:1510.06690].

[36] M. V. Garzelli, S. Moch, and G. Sigl, Lepton fluxes from atmospheric charm revisited, JHEP 10 (2015) 115, [arXiv:1507.01570].

[37] N. Kidonakis, E. Laenen, S. Moch, and R. Vogt, Threshold effects in charm hadroproduction, Phys. Rev. D67 (2003) 074037, [hep-ph/ 0212173$].$

[38] C. Lourenco and H. K. Wohri, Heavy flavour hadro-production from fixed-target to collider energies, Phys. Rept. 433 (2006) 127-180, [hep-ph/ 0609101 ].

[39] PHENIX Collaboration, A. Adare et al., Measurement of high- $p(T)$ single electrons from heavy-flavor decays in $p+p$ collisions at $\sqrt{s}=200-G e V$, Phys. Rev. Lett. 97 (2006) 252002, [hep-ex/ 0609010 ].

[40] STAR Collaboration, L. Adamczyk et al., Measurements of $D^{0}$ and $D^{*}$ Production in $p+p$ Collisions at $\sqrt{s}=200$ GeV, Phys. Rev. D86 (2012) 072013, [arXiv: 1204.4244 ].

[41] ALICE Collaboration, B. Abelev et al., Measurement of charm production at central rapidity in proton-proton collisions at $\sqrt{s}=2.76 \mathrm{TeV}$, JHEP 07 (2012) 191, [arXiv:1205.4007].

[42] ATLAS Collaboration, Measurement of $D^{*}$ meson production cross sections in pp collisions at sqrts $=7 \mathrm{TeV}$ with the ATLAS detector, .

[43] S. Alioli, P. Nason, C. Oleari, and E. Re, A general framework for implementing NLO calculations in shower Monte Carlo programs: the POWHEG BOX, JHEP 1006 (2010) 043, [arXiv: 1002.2581 ]. 
[44] T. Sjostrand, S. Mrenna, and P. Z. Skands, PYTHIA 6.4 Physics and Manual, JHEP 0605 (2006) 026, [hep-ph/0603175].

[45] S. Alekhin, J. Blümlein, and S. Moch, Parton Distribution Functions and Benchmark Cross Sections at NNLO, Phys.Rev. D86 (2012) 054009, [arXiv: 1202.2281].

[46] PROSA Collaboration, O. Zenaiev et al., Impact of heavy-flavour production cross sections measured by the LHCb experiment on parton distribution functions at low x, Eur. Phys. J. $\mathbf{C 7 5}$ (2015), no. 8 396, [arXiv:1503.04581].

[47] ZEUS, H1 Collaboration, F. D. Aaron et al., Combined Measurement and QCD Analysis of the Inclusive e+- p Scattering Cross Sections at HERA, JHEP 01 (2010) 109, [arXiv: 0911.0884 ].

[48] NNPDF Collaboration, R. D. Ball et al., Parton distributions for the LHC Run II, JHEP 1504 (2015) 040, [arXiv:1410.8849].

[49] R. Gauld, J. Rojo, L. Rottoli, and J. Talbert, Charm production in the forward region: constraints on the small-x gluon and backgrounds for neutrino astronomy, JHEP 11 (2015) 009, [arXiv:1506.08025].

[50] S. Alekhin, J. Blümlein, and S. Moch, The ABM parton distributions tuned to LHC data, Phys.Rev. D89 (2014), no. 5 054028, [arXiv: 1310 . 3059].

[51] P. Lipari, Lepton spectra in the earth's atmosphere, Astropart.Phys. 1 (1993) 195-227.

[52] M. Honda, T. Kajita, K. Kasahara, S. Midorikawa, and T. Sanuki, Calculation of atmospheric neutrino flux using the interaction model calibrated with atmospheric muon data, Phys. Rev. D75 (2007) 043006, [astro-ph/0 611418].

[53] IceCube Collaboration, M. Aartsen et al., Evidence for High-Energy Extraterrestrial Neutrinos at the IceCube Detector, Science 342 (2013) 1242856, [arXiv: 1311.5238 ].

[54] IceCube Collaboration Collaboration, M. Aartsen et al., Observation of High-Energy Astrophysical Neutrinos in Three Years of IceCube Data, Phys.Rev.Lett. 113 (2014) 101101,

[arXiv:1405.5303].

[55] T. Sjostrand, S. Mrenna, and P. Z. Skands, A Brief Introduction to PYTHIA 8.1, Comput. Phys. Commun. 178 (2008) 852-867, [arXiv:0710 . 3820].

[56] R. Gauld, J. Rojo, L. Rottoli, S. Sarkar, and J. Talbert, The prompt atmospheric neutrino flux in the light of LHCb, arXiv: 1511.06346.

[57] T. K. Gaisser, T. Stanev, and S. Tilav, Cosmic Ray Energy Spectrum from Measurements of Air Showers, Front.Phys.China 8 (2013) 748-758, [arXiv: 1303.3565$].$

[58] T. Stanev, T. K. Gaisser, and S. Tilav, High energy cosmic rays: sources and fluxes, Nucl.Instrum.Meth. A742 (2014) 42-46.

[59] P. Gondolo, G. Ingelman, and M. Thunman, Charm production and high-energy atmospheric muon and neutrino fluxes, Astropart.Phys. 5 (1996) 309-332, [hep-ph/9505417].

[60] R. Enberg, M. H. Reno, and I. Sarcevic, Prompt neutrino fluxes from atmospheric charm, Phys.Rev. D78 (2008) 043005, [arXiv: 0806.0418 ].

[61] A. Bhattacharya, R. Enberg, M. H. Reno, I. Sarcevic, and A. Stasto, Perturbative charm production and the prompt atmospheric neutrino flux in light of RHIC and LHC, arXiv:1502.01076.

[62] A. Fedynitch, R. Engel, T. K. Gaisser, F. Riehn, and T. Stanev, Calculation of conventional and prompt lepton fluxes at very high energy, EPJ Web Conf. 99 (2015) 08001, [arXiv: 1503.00544 ]. 